\title{
REFLECTION PRINCIPLE FOR SOLUTIONS OF ELLIPTIC PARTIAL DIFFERENTIAL EQUATIONS AND QUASIREGULAR MAPPINGS
}

\author{
O. MARTIO
}

\section{Introduction}

Let $G$ be a domain in the upper half plane $H^{+}$and $C \subset \partial H^{+}$an open set in $\partial G$. If $f=u+i v: G \rightarrow \mathbf{C}$ is analytic and $\lim _{x \rightarrow y} v(x)=0$ for all $y \in C$, it is well known that $f$ has an analytic extension to $G \cup C \cup P G$ where $P$ is the reflection in $\partial H^{+}$. A similar principle holds for harmonic functions in $R^{n}, n \geqq 2$, and for $n=2$ these two principles are intimately connected. In this paper these principles are extended to general non-linear elliptic partial differential equations in divergence form

$$
\nabla \cdot A(x, \nabla u)=0
$$

and to quasiregular mappings, generalizations of plane analytic functions to higher dimensional euclidean spaces. The mean value property is used to prove the reflection principle in the classical harmonic case. No such method is available in the general case and hence different tools are employed. One of the problems is the reflection of the equation (1.1), since if $u$ is a solution of (1.1), then the reflected function should be a solution of the reflected equation $\nabla \cdot A^{*}(x, \nabla u)=0$. This leaves little choice for $A^{*}$, and it turns out that $A$ and $A^{*}$ are quite similar.

For linear and analytic partial differential equations the reflection principle has been extensively studied, see e.g. [J]. For quasiregular mappings it has been earlier treated in $[M R]$ in a special case.

The paper is organized as follows. After preliminaries and a lemma on local integrability of solutions up to the boundary the reflection principle for (1.1) is considered in Chapter 3. Chapter 4 is devoted to the corresponding result for quasiregular mappings.

doi:10.5186/aasfm.1981.0610 


\section{Preliminary lemma}

2.1. Sobolev space $W_{p}^{\mathbf{1}}$. The open ball centered at $x \in R^{n}$ with radius $r>0$ is denoted by $B^{n}(x, r)$ and its boundary by $S^{n-1}(x, r)$. We use the abbreviations $B^{n}(r)=B^{n}(0, r), B^{n}=B^{n}(1), S^{n-1}(r)=S^{n-1}(0, r)$, and $S^{n-1}=S^{n-1}(1)$.

Given an open set $G \subset R^{n}, W_{p}^{1}(G), 1<p<\infty$, denotes the Sobolev space of all functions $u \in L^{p}(G)$ with distributional first derivatives in $L^{p}(G)$, see [GT]. The space $W_{p, 0}^{\mathbf{1}}(G)$ is the closure in $W_{p}^{\mathbf{1}}(G)$ of $C_{0}^{\infty}(G)$-functions. A function $u$ belongs to $\operatorname{loc} W_{p}^{1}(G)$ if for all domains $D \subset \subset G, u \mid D \in W_{p}^{1}(D)$. It is well known that a continuous function $u$ on $G$ belongs to $\operatorname{loc} W_{p}^{1}(G)$ if and only if $u$ is $\mathrm{ACL}^{p}$ in $G$. In our terminology, $\mathrm{ACL}^{p}$ means the set of all continuous (real valued) functions on $G$ which are absolutely continuous on almost every line in $G$ with partial derivatives locally $L^{p}$-summable, see e.g. [GT, p. 163]. The $L^{p}$-norm on $G$ is written as \|\|$_{p, G}$ or simply \|\|$_{p}$.

We shall frequently use the following lemma which is difficult to locate in the literature.

2.2. Lemma. Let $G$ be a bounded open set in $R^{n}$. If $u \in C(\bar{G}) \cap W_{p}^{1}(G)$ and $u=0$ in $\partial G$, then $u \in W_{p, 0}^{1}(G)$.

Proof. Consider the measures

$$
\mu(A)=\int_{A}|u|^{p} d m, \quad \sigma(A)=\int_{A}|\nabla u|^{p} d m
$$

defined on measurable subsets of $A$. Using the $\mathrm{ACL}^{p}$-property it is easy to see that $\sigma$ is absolutely continuous with respect to $\mu$. For $\varepsilon>0$ consider the function $v_{\varepsilon}=\max (u, \varepsilon)-\varepsilon$. Then $v_{\varepsilon} \in W_{p}^{1}(G) \cap C_{0}(G)$. Choose a convolution approximation $\varphi \in C_{0}^{\infty}(G)$ for $v_{\varepsilon}$ such that

If $u^{+}=\max (u, 0)$, then

$$
\left\|\varphi-v_{\varepsilon}\right\|_{p}+\left\|\nabla \varphi-\nabla v_{\varepsilon}\right\|_{p}<\varepsilon
$$

$$
\begin{aligned}
& \left\|u^{+}-\varphi\right\|_{p}+\left\|\nabla u^{+}-\nabla \varphi\right\|_{p}<\left\|u^{+}-v_{\varepsilon}\right\|_{p}+\left\|\nabla u^{+}-\nabla v_{\varepsilon}\right\|_{p}+\varepsilon \\
& \leqq \varepsilon m(G)^{1 / p}+\left(\int_{0 \leqq u(x) \leqq \varepsilon}|\nabla u|^{p} d m\right)^{1 / p}+\varepsilon .
\end{aligned}
$$

The last integral can be made small by the absolute continuity of $\sigma$ since

$$
\mu(\{x \in G: 0 \leqq u(x) \leqq \varepsilon\}) \leqq \varepsilon^{p} m(G) .
$$

A similar estimate holds for $u^{-}=\min (u, 0)$. This completes the proof.

2.3. Elliptic equations. Let $G$ be an open set in $R^{n}$. We consider an elliptic partial equation in divergence form

$$
\nabla \cdot A(x, \nabla u)=0
$$


where $A: G \times R^{n} \rightarrow R^{n}$ is a Borel function such that for a.e. $x \in G$ and for all $h \in R^{n}$

$$
\begin{aligned}
|A(x, h)| & \leqq \gamma|h|^{p-1}, \\
A(x, h) \cdot h & \geqq \alpha|h|^{p} .
\end{aligned}
$$

Here $1<p<\infty$ and $0<\alpha, \gamma<\infty$. An $\mathrm{ACL}^{p}$-function $u: G \rightarrow R$ is a solution of (2.4) if for all $\varphi \in C_{0}^{\infty}(G)$

$$
\int_{G} A(x, \nabla u) \cdot \nabla \varphi d m=0 .
$$

It is well known that the continuity is superfluous in the definition of a solution, since every function $u \in \operatorname{loc} W_{p}^{1}(G)$ satisfying (2.7) can be made continuous after a change on a set of measure zero, see e.g. [GT].

The next lemma gives a local integrability result up to the boundary. Let $G$ and $H$ be open sets in $R^{n}$. Suppose that $E=H \cap \partial G \neq \emptyset$ and write $G^{\prime}=H \cap G$.

2.8. Lemma. Suppose that $h \in C\left(\bar{G}^{\prime}\right) \cap W_{p}^{1}\left(G^{\prime}\right)$. Let $u$ be a solution of the equation (2.4) in G. If $\lim _{x \rightarrow y} u(x)=h(y)$ for all $y \in E$, then each $y \in E$ has a neighborhood $U$ such that $u$ belongs to $W_{p}^{1}(G \cap U)$.

Proof. Fix $y \in E$. We may assume that $y=0$. Choose $\varrho>0$ so that $\bar{B}^{n}(\varrho) \subset H$. Set $B=B^{n}(\varrho) \cap G$. Let $r=\varrho / 2$ and $\varepsilon>0$. Now $u-\varepsilon$ is a solution of (2.4) in $G$. Let $\varphi \in C_{0}^{\infty}\left(B^{n}(\varrho)\right)$ be such that $0 \leqq \varphi \leqq 1$ and $\varphi \mid B^{n}(r)=1$. Consider the function $v=\varphi^{p}(u-\varepsilon-h)$ in the open set $D_{\varepsilon}=\{x \in B: u(x)>\varepsilon+h(x)\}$. Now $D_{\varepsilon} \subset \subset G$ and by Lemma 2.2, $v \in W_{p, 0}^{1}\left(D_{\varepsilon}\right)$. From (2.7) it follows that

$$
\int_{D_{\varepsilon}} A(x, \nabla u) \cdot \nabla v d m=0 .
$$

Since $\nabla v=p \varphi^{p-1} \nabla \varphi(u-\varepsilon-h)+\varphi^{p} \nabla(u-\varepsilon-h)$, Schwarz's inequality yields

$$
\begin{aligned}
& \int_{D_{\varepsilon}} \varphi^{p} A(x, \nabla u) \cdot \nabla u d m \leqq p \int_{D_{\varepsilon}} \varphi^{p-1}|A(x, \nabla u)||\nabla \varphi||u-\varepsilon-h| d m \\
& +\int_{D_{\varepsilon}} \varphi^{p}|A(x, \nabla u)||\nabla h| d m \\
& \leqq M p \gamma \int_{D_{\varepsilon}} \varphi^{p-1}|\nabla u|^{p-1}|\nabla \varphi| d m+\gamma \int_{D_{\varepsilon}} \varphi^{p}|\nabla u|^{p-1}|\nabla h| d m \\
& \leqq C^{\prime}\|\varphi \mid \nabla u\|_{p, D_{\varepsilon}}^{p-1}\left(\|\nabla \varphi\|_{p}+\|\varphi|\nabla h|\|_{p}\right) .
\end{aligned}
$$

Here Hölder's inequality has been used in the last step, $M=\sup _{B}|u-\varepsilon-h|$, and $C^{\prime}$ does not depend on $\varepsilon<1$. Since by (2.6)

$$
\int_{D_{\varepsilon}} \varphi^{p} A(x, \nabla u) \cdot \nabla u d m \geqq \alpha \int_{D_{\varepsilon}} \varphi^{p}|\nabla u|^{p} d m,
$$

the inequality (2.9) yields

$$
\int_{B^{n}(r) \cap D_{\varepsilon}}|\nabla u|^{p} d m \leqq \int_{D_{\varepsilon}} \varphi^{p}|\nabla u|^{p} d m \leqq\left[C^{\prime} \alpha^{-1}\left(\|\nabla \varphi\|_{p}+\|\nabla h\|_{p}\right)\right]^{p}=C^{\prime \prime}
$$


where $C^{\prime \prime}<\infty$ does not depend on $\varepsilon$. Letting $\varepsilon \rightarrow 0$ gives

$$
\int_{B^{n}(r) \cap D_{0}}|\nabla u|^{p} d m<\infty .
$$

A similar estimate holds in the set $B^{n}(r) \cap\{x \in G: u(x)<h(x)\}$. Since

we have proved

$$
\int_{u(x)=h(x)}|\nabla u|^{p} d m=\int_{u(x)=h(x)}|\nabla h|^{p} d m,
$$

$$
\int_{B^{n}(r) \cap G}|\nabla u|^{p} d m<\infty .
$$

By continuity $u \in L^{p}\left(B^{n}(r) \cap G\right)$, thus $u \in W_{p}^{1}\left(B^{n}(r) \cap G\right)$, and the lemma follows.

\section{Reflection principle for solutions of elliptic equations}

Consider the equation (2.4) where $A$ satisfies (2.5) and (2.6) in an open set $G \subset H^{+}=\left\{x \in R^{n}: x_{n}>0\right\}$. Moreover, we assume that for a.e. $x \in G$

$$
A(x,-h)=-A(x, h)
$$

for all $h \in R^{n}$. Let $P: R^{n} \rightarrow R^{n}$ be the reflection in $\partial H^{+}$, i.e. $P(x)=\left(x_{1}, \ldots, x_{n-1},-x_{n}\right)$. Suppose that there is a non-empty set $C \subset \partial H^{+}$open in $\partial G$. Set $G^{*}=G \cup C \cup P G$. Then $G^{*}$ is an open set in $R^{n}$.

Next we reflect the equation (2.4). Define $A^{*}: G^{*} \times R^{n} \rightarrow R^{n}$ as follows

$$
A^{*}(x, h)= \begin{cases}A(x, h), & x \in G \\ 0, & x \in C \\ P A(P(x), P h), & x \in P G,\end{cases}
$$

whenever $h \in R^{n}$. The reflection $P$ is treated as a linear map of $R^{n}$. Observe that $P$ is self-adjoint and $P P=\mathrm{id}$.

Note the following simple but important fact. Since for $x \in P G$,

and

$$
\left|A^{*}(x, h)\right|=|A(P(x), P h)| \leqq \gamma|P h|^{p-1}=\gamma|h|^{p-1}
$$

$$
A^{*}(x, h) \cdot h=A(P(x), P h) \cdot P h \geqq \alpha|P h|^{p}=\alpha|h|^{p},
$$

the function $A^{*}$ satisfies the same assumptions (2.5), (2.6), and (3.1) in $G^{*}$ as $A$ satisfies in $G$.

3.2. Remark. If $F: G \times R^{n} \rightarrow R$ is the variational kernel $F(x, h)=|h|^{p}, p>1$, then the Euler equation corresponding to the variational integral

$$
\int_{G} F(x, \nabla u) d m
$$


takes the form

$$
\nabla \cdot\left(|\nabla u|^{p-2} \nabla u\right)=0
$$

and hence $A(x, h)=|h|^{p-2} h$. Thus $A$ satisfies (2.5), (2.6), and (3.1). Moreover, $A^{*}(x, h)=A(x, h)$. The case $p=2$ is the classical harmonic case. Note that if $A(x, h)=f(|h|) h$ and $f$ is real valued, then $A^{*}(x, h)=A(x, h)$.

Suppose now that $G \subset H^{+}$and $C$ are as above. Let $u$ be a solution of $\nabla \cdot A(x, \nabla u)=0$ in $G$ where $A$ satisfies (2.5), (2.6), and (3.1) and

$$
\lim _{x \rightarrow y} u(x)=0
$$

for all $y \in C$. Define $u^{*}: G^{*} \rightarrow R$ by

$$
u^{*}(x)= \begin{cases}u(x), & x \in G, \\ 0, & x \in C, \\ -u(P(x)), & x \in P G .\end{cases}
$$

3.4. Theorem. The function $u^{*}$ is a solution of the equation $\nabla \cdot A^{*}(x, \nabla v)=0$ in $G^{*}$.

Proof. By (3.3) the function $u^{*}$ is continuous in $G^{*}$. By Lemma 2.8 each point $y \in C$ has a neighborhood $U$ such that $u$ belongs to $W_{p}^{1}\left(U \cap H^{+}\right)$and $U=P U$. Hence $u^{*}$ also belongs to $W_{p}^{1}\left(U \cap H^{-}\right), H^{-}=R^{n} \backslash \bar{H}^{+}$, and the continuity of $u^{*}$ implies that $u^{*}$ is $\mathrm{ACL}^{p}$ in $U$. Thus $u^{*}$ is $\mathrm{ACL}^{p}$ in $G$ and it remains to show that $u^{*}$ satisfies

$$
\nabla \cdot A^{*}(x, \nabla v)=0
$$

in $G^{*}$. Since this problem is local it suffices to show that (i) $u^{*}$ is a solution of (3.5) in $P G$ and that (ii) each point $y \in C$ has a neighborhood $U$ such that $u^{*}$ is a solution of (3.5) in $U$.

We first prove (i). Let $\varphi \in C_{0}^{\infty}(P G)$. Now $\nabla u^{*}(x)=-P \nabla u(P(x))$ for a.e. $x \in P G$, and since the Jacobian determinant of $P$ satisfies $|J(x, P)|=1$, the integral transformation formula yields

$$
\begin{aligned}
& \int_{P G} A^{*}\left(x, \nabla u^{*}\right) \cdot \nabla \varphi d m=\int_{P G} P A(P(x),-\nabla u(P(x))) \cdot \nabla \varphi(x) d m \\
& =-\int_{P G} A(P(x), \nabla u(P(x))) \cdot P \nabla(\varphi(P P(x)))|J(x, P)| d m \\
& =-\int_{G} A(x, \nabla u(x)) \cdot P \nabla(\varphi(P(x))) d m=-\int_{G} A(x, \nabla u) \cdot \nabla \psi d m=0,
\end{aligned}
$$

where $\psi=\varphi \circ P \in C_{0}^{\infty}(G)$ and the assumption (3.1) has also been used. Thus $u^{*}$ is a solution of (3.5) in $P G$. 
To prove (ii) requires more computation. Fix a neighborhood $U$ of $y \in C$ such that $U=P U$ and $U \subset G^{*}$. For $t>0$ let $K_{t}=\left\{x \in R^{n}:\left|x_{n}\right| \leqq t\right\}$ and choose $h_{t} \in C^{\infty}\left(R^{n}\right)$ such that (a) $0 \leqq h_{t} \leqq 1$, (b) $h_{t} \mid K_{t / 2}=1$, (c) $h_{t} \mid R^{n} \backslash K_{t}=0$, (d) $\left|\nabla h_{t}\right| \leqq c / t$ where $c$ is a constant, and (e) $h(x)=h(P(x))$.

Let $\varphi \in C_{0}^{\infty}(U)$ be arbitrary. Then

$$
\begin{aligned}
& \int_{U} A^{*}\left(x, \nabla u^{*}\right) \cdot \nabla \varphi d m \\
& =\int_{U} A^{*}\left(x, \nabla u^{*}\right) \cdot \nabla\left(\left(1-h_{t}\right) \varphi\right) d m+\int_{U} A^{*}\left(x, \nabla u^{*}\right) \cdot \nabla\left(h_{t} \varphi\right) d m .
\end{aligned}
$$

Now $\left(1-h_{t}\right) \varphi \in C_{0}^{\infty}(G \cup P G)$ and since $u^{*}$ is a solution in $G \cup P G$, the first integral on the right hand side of (3.6) is $=0$. We shall show that the second integral tends to 0 as $t \rightarrow 0$. This will prove (ii). First observe that

$$
\begin{aligned}
& \int_{U} A^{*}\left(x, \nabla u^{*}\right) \cdot \nabla\left(h_{t} \varphi\right) d m \\
& =\int_{U \cap G} h_{t} A(x, \nabla u) \cdot \nabla \varphi d m-\int_{U \cap P G} h_{t} A(P(x), \nabla u(P)) \cdot P \nabla \varphi d m \\
& +\int_{U \cap G} \varphi A(x, \nabla u) \cdot \nabla h_{t} d m-\int_{U \cap P G} \varphi A(P(x), \nabla u(P)) \cdot P \nabla h_{t} d m \\
& =I_{1}-I_{2}+I_{3}-I_{4} .
\end{aligned}
$$

We estimate the differences $I_{1}-I_{2}$ and $I_{3}-I_{4}$ separately.

Write $A_{t}=U \cap K_{t} \cap H^{+}$. For $I_{1}-I_{2}$ we have the estimate

$$
\left|I_{1}-I_{2}\right| \leqq\left|I_{1}\right|+\left|I_{2}\right| \leqq \int_{A_{t}}|A(x, \nabla u)||\nabla \varphi| d m+\int_{P A_{t}}\left|A^{*}\left(x, \nabla u^{*}\right)\right||\nabla \varphi| d m .
$$

Since $u^{*} \in W_{p}^{1}(U)$, Hölder's inequality, (2.5) for $A^{*}$, and $m\left(U \cap K_{t}\right) \rightarrow 0$ as $t \rightarrow 0$ yield $\quad I_{1}-I_{2} \rightarrow 0$ as $t \rightarrow 0$.

To estimate $I_{3}-I_{4}$ we perform a change of variable in $I_{4}$. Since $h_{t}$ is symmetric and hence $\nabla h_{t}=P \nabla h_{t}(P)$, we have

$$
\begin{aligned}
I_{4} & =\int_{U \cap P G} \varphi(P P(x)) A(P(x), \nabla u(P(x))) \cdot P \nabla h_{t}(P P(x))|J(x, P)| d m \\
& =\int_{U \cap G} \varphi(P(x)) A(x, \nabla u(x)) \cdot P \nabla h_{t}(P(x)) d m \\
& =\int_{U \cap G} \varphi(P(x)) A(x, \nabla u(x)) \cdot \nabla h_{t}(x) d m .
\end{aligned}
$$


Thus by (d) and (2.5)

$$
\begin{aligned}
& \left|I_{3}-I_{4}\right| \leqq \sup _{K_{t}}|\varphi(x)-\varphi(P(x))| \int_{A_{t}}|A(x, \nabla u)|\left|\nabla h_{t}\right| d m \\
& \leqq c^{\prime} t c t^{-1} \gamma \int_{A_{t}}|\nabla u|^{p-1} d m \leqq c^{\prime} c \gamma\|\nabla u\|_{p, U \cap G}^{(p-1) / p} m\left(A_{t}\right)^{1 / p},
\end{aligned}
$$

since for the Lipschitz-function $\varphi, \sup _{K_{t}}|\varphi(x)-\varphi(P(x))| \leqq c^{\prime} t$. Thus $m\left(A_{t}\right) \rightarrow 0$ yields $I_{3}-I_{4} \rightarrow 0$ as $t \rightarrow 0$. This proves (ii) and Theorem 3.4 follows.

\section{Reflection principle for quasiregular mappings}

A vector valued $\mathrm{ACL}^{n}$-mapping $f: G \rightarrow R^{n}$, where $G \subset R^{n}$ is a domain, is called quasiregular if for some $K \geqq 1$

$$
\left|f^{\prime}(x)\right|^{n} \leqq K J(x, f)
$$

for a.e. $x \in G$. Here $\left|f^{\prime}(x)\right|$ means the sup-norm of the linear mapping $f^{\prime}(x): R^{n} \rightarrow R^{n}$. The smallest $K$ for which (4.1) holds is denoted by $K_{O}(f)$. If $f$ is quasiregular, then

$$
J(x, f) \leqq K_{I}(f) l\left(f^{\prime}(x)\right)^{n}
$$

a.e. in $G$ and $K_{I}(f) \leqq K_{O}(f)^{n-1}$. Here $l\left(f^{\prime}(x)\right)=\inf \left\{\left|f^{\prime}(x) h\right|:|h|=1\right\}$. The maximal dilatation $K(f)$ of $f$ is the number $\max \left(K_{I}(f), K_{O}(f)\right)$. For the theory of quasiregular mappings we refer to [MRV].

Let $G$ be a domain in the upper half space $H^{+}$and suppose that there is a non-empty set $C \subset \partial H^{+} \cap \partial G$ open in $\partial G$. Then $G^{*}=G \cup C \cup P G$ is a domain in $R^{n}$. Suppose that $f=\left(f_{1}, \ldots, f_{n}\right): G \rightarrow R^{n}$ is a quasiregular mapping. Define a mapping $f^{*}: G \cup P G \rightarrow R^{n}$ by

$$
f^{*}(x)= \begin{cases}f(x), & x \in G \\ P(f(P(x))), & x \in P G .\end{cases}
$$

The extension of the reflection principle of plane analytic functions to quasiregular mappings takes the following form.

4.3. Theorem. Let

$$
\lim _{x \rightarrow y} f_{n}(x)=0
$$

for all $y \in C$. Then $f^{*}$ defines a quasiregular mapping $G^{*} \rightarrow R^{n}$.

4.5. Remark. If $f^{*}$ is quasiregular, it is clear that

$$
K_{o}\left(f^{*}\right)=K_{o}(f) \text { and } K_{I}\left(f^{*}\right)=K_{I}(f) .
$$


To prove Theorem 4.3 we need a counterpart of a well known property of analytic functions. If $f=u+i v$ is analytic in $D$ and $|\nabla v|$ is $L^{p}$-integrable in $D$, then by the Cauchy-Riemann equations $\left|f^{\prime}\right|=|\nabla v|$ and hence $\left\|f^{\prime}\right\|_{p}=\|\nabla v\|_{p}$.

4.6. Lemma. Let $f=\left(f_{1}, \ldots, f_{n}\right)$ be a quasiregular mapping of a domain $D$ into $R^{n}$ and $p>0$. Then for $1 \leqq i \leqq n$

$$
\left(\int_{D}\left|f^{\prime}(x)\right|^{p} d m\right)^{1 / p} \leqq K(f)^{2 / n}\left(\int_{D}\left|\nabla f_{i}\right| d m\right)^{1 / p} .
$$

Proof. For a.e. $x \in G$ and all $i, 1 \leqq i \leqq n$,

$$
\begin{aligned}
\left|f^{\prime}(x)\right| & \leqq K_{o}(f)^{1 / n} J(x, f)^{1 / n} \leqq\left(K_{O}(f) K_{I}(f)\right)^{1 / n} l\left(f^{\prime}(x)\right) \\
& \leqq K(f)^{2 / n}\left|\nabla f_{i}(x)\right|
\end{aligned}
$$

and the result follows by integration.

Proof for Theorem 4.3. The coordinate functions of $f$, and hence $f_{n}$, are solutions of a differential equation $\nabla \cdot A(x, \nabla u)=0$ where $A: G \times R^{n} \rightarrow R^{n}$ satisfies (2.5), (2.6) and (3.1) for $p=n$, cf. [R2]. Let $y \in C$. By (4.4) and Lemma 2.8 there is $r>0$ such that $f_{n} \in W_{n}^{1}\left(H^{+} \cap B^{n}(y, r)\right)$. By Lemma 4.6

$$
\int_{H^{+} \cap B^{n}(y, r)}\left|f^{\prime}(x)\right|^{n} d m<\infty .
$$

This means that the coordinate functions $f_{i}$ of $f$ have derivatives in $L^{n}\left(H^{+} \cap B^{n}(y, r)\right)$. Using the $\mathrm{ACL}^{n}$-property and Fubini's theorem it is easy to see that $f$ has a finite, limit along the line segment $L_{z}=\left\{z+t e_{n}: t>0\right\}$ for almost every $z \in \partial H^{+} \cap B^{n}(y, r)$. Clearly

$$
\int_{H-\cap B^{n}(y, r)}\left|f^{*^{\prime}}(x)\right|^{n} d m<\infty
$$

and $f^{*}$ has the same limit from the opposite direction along $P L_{z}$. Thus $f^{*} \in W_{n}^{1}\left(B^{n}(y, r)\right)$ and $\left|f^{* \prime}(x)\right|^{n} \leqq K_{O}(f) J\left(x, f^{*}\right)$ for a.e. $x \in B^{n}(y, r)$. By a theorem of Rešetnjak [R1, Theorem 1] there is a continuous mapping $g$ such that $g=f^{*}$ a.e. This completes the proof.

4.7. Remark. It is clear that using preliminary Möbius transformations we can formulate the reflection principle as well in a domain $G \subset B^{n}$ with a set $C \subset \partial G \cap$ $S^{n-1}$ open in $\partial G$. The condition (4.4) takes the form $\lim _{x \rightarrow y}|f(x)|=1$ for all $y \in C$ and instead of $P$ the reflection in $S^{n-1}$ is used. There is a way to prove this reflection principle which does not make explicit use of Lemma 4.6. The proof is based on the study of the function $u(x)=\log |f(x)|$ which also satisfies an equation of the form $\nabla \cdot A(x, \nabla v)=0$. Note that $|\nabla u(x)| \geqq\left|f^{\prime}(x)\right|(K(f)|f(x)|)^{-1}$ a.e. and the estimate of Lemma 4.6 can be obtained from the $L^{n}$-integrability of $\nabla u$. 


\title{
References
}

[GT] Gilbarg, D., and N. S. Trudinger: Elliptic partial differential equations of second order. - Springer-Verlag, Berlin-Heidelberg-New York, 1977.

[GLM] Granlund, S., P. Lindqvist, and O. Martio: Conformally invariant variational integrals. To appear.

[J] JoHN, F.: Continuation and reflection of solutions of partial differential equations. - Bull. Amer. Math. Soc. 63, 1957, 327-344.

[MR] Martio, O., and S. Rickman: Boundary behavior of quasi-regular mappings. - Ann. Acad. Sci. Fenn. Ser. A I 507, 1972, 1-17.

[MRV] Martio, O., S. Rickman, and J. VÄIsÄLÄ: Definitions for quasiregular mappings. - Ann. Acad. Sci. Fenn. Ser. A I 448, 1969, 1-40.

[R1] REŠETNJAK, JU. G. (Решетняк, Ю. Г.): Оценки модуля непрерывности для некоторых отображений. - Sibirsk. Mat. Ž. 7, 1966, 1106-1114.

[R2] REŠETNJAK, JU. G. (Решетняк, Ю. Г.): Отображения с ограниченным искажением как экстремали интегралов типа Дирихле. - Sibirsk. Mat. Ž. 9, 1968, 652-666.

\author{
University of Helsinki \\ Department of Mathematics \\ SF-00100 Helsinki 10 \\ Finland
}

Received 19 January 1981 